\title{
Effect of melatonin on incidence of delirium among patients with hip fracture: a multicentre, double-blind randomized controlled trial
}

\author{
Annemarieke de Jonghe MD PhD, Barbara C. van Munster MD PhD, J. Carel Goslings MD PhD, \\ Peter Kloen MD PhD, Carolien van Rees MD, Reinder Wolvius MD, Romuald van Velde MD, \\ Marcel Levi MD PhD, Rob J. de Haan PhD, Sophia E. de Rooij MD PhD; on behalf of the Amsterdam \\ Delirium Study Group*
}

\begin{abstract}
- Abstract
Background: Disturbance of the sleep-wake cycle is a characteristic of delirium. In addition, changes in melatonin rhythm influence the circadian rhythm and are associated with delirium. We compared the effect of melatonin and placebo on the incidence and duration of delirium.

Methods: We performed this multicentre, double-blind, randomized controlled trial between November 2008 and May 2012 in 1 academic and 2 nonacademic hospitals. Patients aged 65 years or older who were scheduled for acute hip surgery were eligible for inclusion. Patients received melatonin $3 \mathrm{mg}$ or placebo in the evening for 5 consecutive days, starting within 24 hours after admission. The primary outcome was incidence of delirium within 8 days of admission. We also monitored the duration of delirium.

Results: A total of 452 patients were randomly assigned to the 2 study groups. We subsequently excluded 74 patients for whom the pri-

mary end point could not be measured or who had delirium before the second day of the study. After these postrandomization exclusions, data for 378 patients were included in the main analysis. The overall mean age was 84 years, $238(63.0 \%)$ of the patients lived at home before admission, and 210 (55.6\%) had cognitive impairment. We observed no effect of melatonin on the incidence of delirium: 55/186 $(29.6 \%)$ in the melatonin group v. $49 / 192$ $(25.5 \%)$ in the placebo group; difference 4.1 ( $95 \%$ confidence interval -0.05 to 13.1 ) percentage points. There were no between-group differences in mortality or in cognitive or functional outcomes at 3-month follow-up.

Interpretation: In this older population with hip fracture, treatment with melatonin did not reduce the incidence of delirium. Trial registration: Netherlands Trial Registry, NTR1576: MAPLE (Melatonin Against PLacebo in Elderly patients) study; www.trialregister.nl /trialreg/admin/rctview.asp?TC=1576
\end{abstract}

$\mathrm{D}$ elirium in older inpatients is associated with a high risk of dementia and other complications that translate into increased mortality and health care costs. ${ }^{1,2}$ The antipsychotic haloperidol has historically been the agent of choice for treating delirium, and it has increasingly been administered as a prophylactic for delirium or to reduce symptoms such as hallucinations and aggressive behaviour. ${ }^{3,4}$ However, all antipsychotic treatments may induce serious cerebrovascular adverse effects and greater mortality, particularly among patients with dementia. ${ }^{5,6}$ These effects led the US Food and Drug Administration to issue a serious warning against their use. ${ }^{7}$ In addition, benzodiazepines are still frequently used to treat delirium, despite their being known to elicit or aggravate delirium. ${ }^{8,9}$
Disturbances of the circadian sleep-wake cycle represent one of the core features of delirium, ${ }^{10}$ leading to the hypothesis that the neurotransmitter melatonin and changes in its metabolism may be involved in the pathogenesis of delirium. ${ }^{11,12}$ Objective measurements have shown that melatonin metabolism is disturbed after abdominal and other types of surgery, insomnia, sleep deprivation and stays in the intensive care unit (ICU), all of which are also known to be factors that contribute to delirium. ${ }^{13-16}$ These characteristics suggest an association between melatonin abnormalities and delirium. ${ }^{17-22}$ Although proof of a causal relation is still lacking, inpatients might nevertheless benefit from melatonin supplementation therapy through postoperative maintenance or restoration of their sleep-wake cycle. ${ }^{23-25}$ Although melato-
Competing interests: None declared.

This article has been peer reviewed.

*The list of members of the Amsterdam Delirium Study Group appears at the end of the article.

Correspondence to: Annemarieke de Jonghe, a.dejonghe@amc.uva.nl

CMAJ 2014. DOI:10.1503 /cmaj.140495 
nin depletion is thought to be one of the mechanisms of delirium, few studies have investigated the effects of altering perioperative plasma concentrations of melatonin, in particular, the possible effects on postoperative delirium.

The primary objective of this study was to assess the effects of melatonin on the incidence of delirium among elderly patients admitted to hospital as an emergency following hip fracture. Secondary outcomes were duration and severity of delirium, length of hospital stay, total doses of haloperidol and benzodiazepines administered to patients with delirium, mortality during the hospital stay, and functional status, cognitive function and mortality at 3-month follow-up.

\section{Methods}

\section{Trial design}

We conducted this multicentre, double-blind, randomized controlled trial in the Netherlands between November 2008 and May 2012. Full details of the study protocol have been presented elsewhere. ${ }^{26} \mathrm{We}$ conducted the study in compliance with the Declaration of Helsinki and Good Clinical Practice guidelines. ${ }^{27}$ The study was approved by the Medical Ethics Committee of the Academic Medical Center, University of Amsterdam, with local approval from the other participating centres. Written informed consent was obtained from each patient or, for patients with cognitive impairment, from a legal representative. The study had no data safety or monitoring board because melatonin was considered safe. ${ }^{28,29} \mathrm{We}$ reported all serious adverse events to the Medical Ethics Committee and had a protocol to break the randomization code in case of a suspected unexpected serious adverse reaction. This trial was registered with the Netherlands Trial Registry (NTR1576) and was funded by an unrestricted grant (no. 311020301) from the Dutch National Program of Innovative Care for vulnerable older persons (a program operated by ZonMw, a Dutch institute that funds health research and stimulates the use of knowledge to help improve health and health care in the Netherlands).

\section{Participants and setting}

The study population consisted of patients aged 65 years or older who were admitted for emergent surgical treatment of hip fracture. There was no restriction on the type of treatment planned (internal fixation, hemi-arthroplasty or total hip replacement). Patients had to be enrolled within 24 hours after admission and had to be willing and able to receive the study medication for the duration of the study (according to the protocol). Patients who were taking psychiatric medications (i.e., other than medication for delirium) could continue their prescriptions throughout the study period. We excluded patients if they had delirium at the time of enrolment, if they had been transferred from another hospital, if postoperative admission to the ICU or coronary care unit was anticipated (which would create logistic difficulties for conducting the study), if they were already taking melatonin or if they were unable to speak or understand Dutch. The study was conducted in the surgical, orthopedic and trauma surgery wards of the Academic Medical Center (a 1000-bed university teaching hospital in Amsterdam) and the Tergooi Hospitals (a regional teaching hospital comprising 633 beds at 2 locations, Hilversum and Blaricum). An experienced team of geriatric nurses conducted followup visits 3 months after initial enrolment.

\section{Randomization and blinding}

After baseline assessment, we randomly assigned each patient to either the intervention group (melatonin) or the control group (placebo) at a 1:1 ratio. Randomization was stratified by study centre, with fixed blocks of 10 patients within each stratum. Before the start of the study, an independent statistician generated a randomization schedule, and the trial pharmacist maintained the randomization list. Study medication tablets were manufactured and labelled according to guidelines for good manufacturing practices. ${ }^{30}$ Investigators, other staff members and patients remained blinded until after the last patient had completed the study and the 3-month follow-up and data analyses had been completed.

\section{Procedures}

All patients aged 65 years or older with emergent hip fracture were approached within 24 hours of admission by a member of our research team, which consisted of geriatricians and trained research nurses with experience in geriatrics. Patients were screened for eligibility and asked to participate. After randomization (as described above), patients received the study medication on 5 consecutive evenings, starting the day of admission, at about $9 \mathrm{pm}$. The study medication consisted of a tablet containing either melatonin $3 \mathrm{mg}$ ( $\pm 5 \%$, i.e., $2.85-3.15 \mathrm{mg}$ per tablet) (Tiofarma, Oud-Beijerland, The Netherlands) or placebo. Surgery usually took place on day 1 or 2 of the admission, in accordance with national quality indicators.

At baseline, we recorded demographic data, medical history, medication use and surgeryrelated characteristics. We assessed functional status with the 15-item modified Katz Index of Activities of Daily Living, ${ }^{31}$ based on the 2 weeks 
before admission. This instrument was completed by the patient or, in cases of cognitive impairment, by the his or her closest relative. We calculated functional impairment as the sum of activities of daily living with impairment. ${ }^{31} \mathrm{We}$ assessed cognitive function with the Mini-Mental State Examination, ${ }^{32}$ a validated 30-point questionnaire. We asked primary caregivers to complete the Informant Questionnaire on Cognitive Decline - short form by recalling the 2 weeks before the hip fracture and comparing this period with 10 years earlier. ${ }^{33,34}$ We defined cognitive impairment as a score of 3.4 or higher on this questionnaire or a record of dementia in the medical history. ${ }^{35}$ We scored the number and severity of comorbidities with the Charlson comorbidity index. ${ }^{36} \mathrm{We}$ asked patients whether they had ever experienced an episode of delirium and whether they had had 2 falls (or more) within the past 3 months. We recorded the total amounts of haloperidol and other antipsychotics and of benzodiazepines that were administered for delirium during the study period. Different types of benzodiazepines were used for this purpose, so we present the amounts as oxazepam equivalents (calculated using a Dutch online conversion tool). Patients with delirium received perioperative care as usual, based on the hospital's routine delirium protocol, as described elsewhere. ${ }^{26}$ At discharge, fracture characteristics, type of anesthesia, type of surgery and length of stay were registered for all patients; we obtained these data, as well as information about in-hospital deaths, from the medical notes. At 3-month follow-up, the visiting nurse performed a Mini-Mental State Examination and asked the patient or closest relative to complete the Katz Index of Activities of Daily Living.

\section{Outcomes}

The primary outcome was incidence of delirium during the first 8 days after initiation of the study medication. We used criteria from the Diagnostic and Statistical Manual of Mental Disorders, fourth edition (DSM-IV), to diagnose delirium. ${ }^{37}$ Over the 8-day period (or until discharge, if the patient was discharged before 8 days), each patient was assessed daily for the presence of delirium; this assessment incorporated all patient information from sources such as medical and nursing records for the previous 24 hours. In addition, the Delirium Observation Screening Scale $^{38}$ was completed for each patient during each nursing shift. For patients who did not have delirium on day 8 , these daily assessments were terminated. For patients who had delirium on day 8 , daily clinical assessments were continued (until the symptoms resolved or until the patient was discharged) to determine the duration of delirium. Secondary outcomes were duration of delirium (total number of days and percentage of patients with duration longer than 2 days), severity of delirium (percentage of patients who received a total of $\geq 3 \mathrm{mg}$ haloperidol), length of hospital stay, total doses of haloperidol and of benzodiazepines (in oxazepam equivalents) administered during delirium (for those patients who experienced delirium), in-hospital mortality rate, and functional status, cognitive function and mortality rate at 3-month follow-up. Cognitive impairment was deemed to be present at 3-month follow-up if dementia was scored in the Charlson comorbidity index and/or the baseline score for the Informant Questionnaire on Cognitive Decline was 3.4 or above and/or the MiniMental State Examination score was below 24 points at 3-month follow-up.

\section{Sample size}

We calculated our sample size from the estimated incidence of delirium during the first 8 days after the start of study medication. At the Academic Medical Center (one of the study centres), the incidence of delirium after surgical repair of hip fracture was previously reported as about $50 \%{ }^{39}$ Literature on medical interventions to reduce the incidence of delirium is rare, but one study showed an absolute reduction of $13 \% .{ }^{40} \mathrm{~A}$ 2-group $\chi^{2}$ test with 2-sided level of significance of 0.05 would have $80 \%$ power to detect the difference between a control group proportion of 0.50 and a treatment group proportion of 0.37 (odds ratio [OR] 0.587) if the sample size in each group were 226 (452 patients in total).

\section{Statistical analysis}

We analyzed the data according to the intentionto-treat principle and secondarily by per-protocol analysis. We summarized baseline characteristics and outcomes with descriptive statistics. We analyzed the between-group difference in the primary outcome (incidence of delirium during the first 8 days after initiation of the study medication) using a $\chi^{2}$ test. We also analyzed the primary outcome using logistic regression, incorporating the stratification variable (treatment centre) and imbalance of baseline variables into the model. We tested the model's goodness of fit with the Hosmer-Lemeshow statistic. We expressed effect size as an adjusted OR. We analyzed continuous secondary outcome measures using 2-group $t$ tests or Mann-Whitney tests, as appropriate. We performed an additional predefined analysis using data from patients with cognitive impairment at baseline, who may react differently to medication because of existing neurodegeneration dependent on the occurrence 
and duration of delirium. No interim analyses were performed. We considered $p$ values less than 0.05 statistically significant.

\section{Results}

\section{Patient recruitment and baseline data}

Between November 2008 and May 2012, we assessed 850 patients for eligibility within 24 hours of admission. Of the 748 eligible patients, consent was obtained from $452(60.4 \%)$, with consent being provided by substitute decision-makers in 167 (36.9\%) of these cases. After randomization, another 8 patients were excluded because of a logistics failure (we could not determine whether they had received placebo or study medication). Therefore, a total of 444 patients were assigned to one of the treatments: 219 to receive melatonin and 225 to receive placebo.

Following initiation of the study medications, we excluded 33 additional patients in each group because the primary outcome (incidence of delirium within 8 days of admission) could not be measured. Among these 66 patients were 18 patients (4 with cognitive impairment) who withdrew their consent. The main reasons for withdrawal of consent were the patient having second thoughts $(n=8)$ and the substitute decision-maker withdrawing consent $(n=8)$; for 2 patients, the reason was unknown. Six additional patients were transferred to other wards, 2 patients did not undergo the planned surgery, and 8 patients died within 8 days after initiation of the study. Most of these patients were excluded on the first or second day of admission. They would otherwise have been considered not to have delirium, although they might have experienced delirium at a later stage. An additional 31 patients were excluded because we determined that delirium was present at the time of enrolment. Symptoms of delirium became apparent in these patients only several hours after they were enrolled, on the first day of the trial, because the nature of the condition lends itself to diagnostic uncertainty and an observation time of 24 hours is required for certainty.

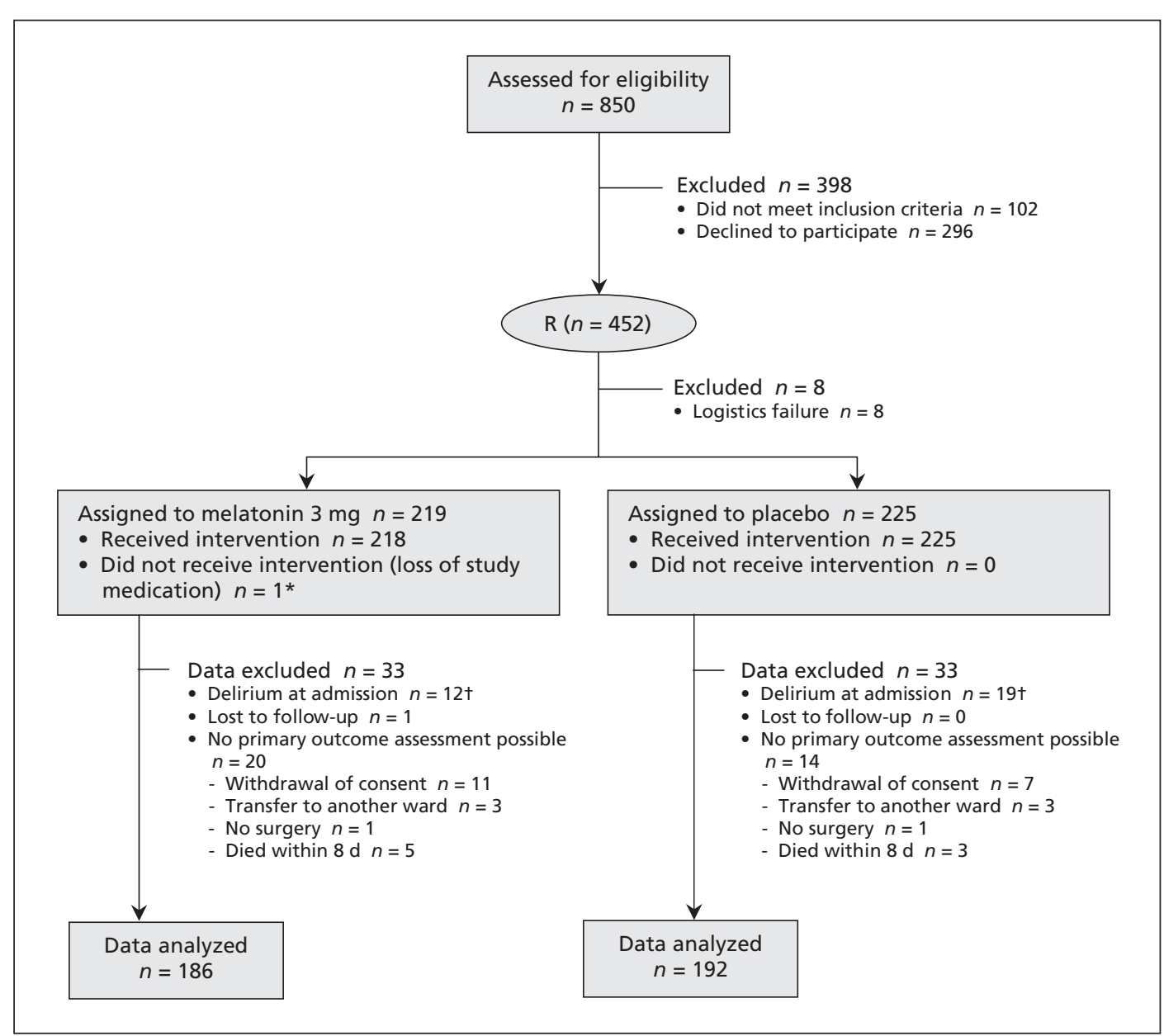

Figure 1: Enrolment, randomization and follow-up of patients. Of the 850 patients assessed for eligibility, 748 met the inclusion criteria and were invited to participate, and 452 (60.4\%) of these provided consent and were randomly assigned to a treatment group. *This patient was included in the analysis. tPatients with delirium at the time of admission were excluded because the primary outcome could not be measured. 
In total, 378 patients were included in the analysis (114 from the Academic Medical Center and 264 from the Tergooi Hospitals). The analysis presented here was based on the initial treatment assignment: 186 patients assigned to receive melatonin and 192 patients assigned to receive placebo (Figure 1). Baseline characteristics were well matched between the 2 study groups,

Table 1: Baseline demographic characteristics of the study patients by treatment group

\begin{tabular}{|c|c|c|}
\hline \multirow[b]{2}{*}{ Characteristic } & \multicolumn{2}{|c|}{ Study group; no. (\%) of patients* } \\
\hline & Melatonin $(n=186)$ & Placebo $(n=192)$ \\
\hline Academic Medical Center & $56(30.1)$ & $58(30.2)$ \\
\hline \multicolumn{3}{|l|}{ Tergooi Hospitals } \\
\hline Hilversum & $85(45.7)$ & $88(45.8)$ \\
\hline Blaricum & $45(24.2)$ & $46(24.0)$ \\
\hline Age, yr, mean \pm SD & $84.1 \pm 8.0$ & $83.4 \pm 7.5$ \\
\hline Sex, male & $53(28.5)$ & $62(32.3)$ \\
\hline Living at home & $116(62.4)$ & $122(63.5)$ \\
\hline Preadmission Katz-ADL score, median (IQR)† & $6(2-10)$ & $5(1-10)$ \\
\hline Data missing & $4 \quad(2.2)$ & $4(2.1)$ \\
\hline MMSE score, median (IQR) & $23(12-28.8)$ & $23(9.5-28.0)$ \\
\hline Data missing & $25(13.4)$ & $32(16.7)$ \\
\hline Cognitive impairment & $104(55.9)$ & $106(55.2)$ \\
\hline Charlson comorbidity index, median (IQR)‡ & $1.0(0.8-2.0)$ & $1.0(1.0-2.0)$ \\
\hline Data missing & $0 \quad(0.0)$ & $0 \quad(0.0)$ \\
\hline \multicolumn{3}{|l|}{ Prior delirium } \\
\hline Yes & $44(23.7)$ & $34(17.7)$ \\
\hline Data missing & $15(8.1)$ & $13(6.8)$ \\
\hline \multicolumn{3}{|l|}{ At least 2 falls in the past $3 \mathrm{mo}$} \\
\hline Yes & $66(35.5)$ & $62(32.3)$ \\
\hline Data missing & $4(2.2)$ & $14(7.3)$ \\
\hline \multicolumn{3}{|l|}{ Medications at home } \\
\hline No., median (IQR) & $5.0(2.0-7.0)$ & $4.0(2.0-7.0)$ \\
\hline Benzodiazepines & $18(9.7)$ & $33(17.2)$ \\
\hline Antipsychotics & $12(6.5)$ & $14(7.3)$ \\
\hline Antidepressants & $26(14.0)$ & $34(17.7)$ \\
\hline \multicolumn{3}{|l|}{ Type of fracture } \\
\hline Femoral neck & $88(47.3)$ & $90(46.9)$ \\
\hline Intertrochanteric & $69(37.1)$ & $74(38.5)$ \\
\hline Subtrochanteric & $1(0.5)$ & $0 \quad(0.0)$ \\
\hline Periprosthetic & $1(0.5)$ & $1(0.5)$ \\
\hline Other & $27(14.5)$ & $27(14.1)$ \\
\hline Spinal anesthesia & $55(29.6)$ & $54(28.1)$ \\
\hline \multicolumn{3}{|l|}{ Type of surgery } \\
\hline Internal fixation & $101(54.3)$ & $108(56.2)$ \\
\hline Hip replacement & 70 (37.6) & $74(38.5)$ \\
\hline Other & $15(8.1)$ & $10(5.2)$ \\
\hline \multicolumn{3}{|c|}{$\begin{array}{l}\text { Note: IQR }=\text { interquartile range, Katz-ADL = Katz Index of Activities of Daily Living, }{ }^{31} \text { MMSE = Mini-Mental State Examination, }{ }^{32} \\
\text { SD = standard deviation. } \\
\text { *Unless indicated otherwise. } \\
\text { †The Katz-ADL score ranges from } 0 \text { to } 15, \text { with higher scores indicating more severe functional dependence. } \\
\text { ¥The Charlson comorbidity index ranges from } 1 \text { to } 31 \text {, with higher scores indicating more comorbidities. }\end{array}$} \\
\hline
\end{tabular}


although the percentage of patients using benzodiazepines at home was higher in the placebo group, whereas more patients in the melatonin group had experienced prior delirium (Table 1). The overall mean age was 84 years, $238(63.0 \%)$ of the patients were living at home before admission, and $210(55.6 \%)$ had cognitive impairment.

\section{Outcomes}

This was a negative study, as we observed no effect of melatonin on the incidence of delirium: $55 / 186(29.6 \%)$ for the melatonin group $\mathrm{v}$. $49 / 192(25.5 \%)$ for the placebo group; absolute difference 4.1 (95\% confidence interval [CI] -0.05 to 13.1 ) percentage points (Table 2). Multivariable logistic regression, adjusted for the stratification variable (treatment centre) and baseline imbalances (benzodiazepine use at home and prior delirium), also did not show any treatment effect (OR 1.14, 95\% CI 0.71-1.83; HosmerLemeshow goodness-of-fit test, $p=0.70$ ).

A smaller proportion of patients in the melatonin group than in the placebo group experienced a long-lasting episode of delirium $(>2$ days) $(25.5 \%$ v. $46.9 \% ; p=0.02)$, although the median duration of delirium was the same for the 2 groups (2 days; Figure 2). The severity of delirium, expressed as the proportion of patients receiving $3 \mathrm{mg}$ or more of haloperidol, was similar, and the median length of hospital stay was the same ( $p=0.4$ for both) (Table 2$)$. The median dose of haloperidol administered for delirium was similar in the 2 groups ( $p=0.2$ ), as was the median dose of benzodiazepines, in oxazepam equivalents $(p=0.6)$. The mortality rate during the hospital stay was about $2 \%$ in both groups $(p>0.9)$.

At 3 months, we observed no between-group differences with regard to cognitive or functional outcomes. The median score for the Katz Index of Activities of Daily Living was 9.0 for both groups $(p=0.8)$ (where scores for this index range from 0 to 15 , with higher scores indicating more severe functional dependence). Cognitive impairment was present in $59.2 \%$ of the patients in the melatonin group and $66.0 \%$ in the placebo group ( $p=$ $0.4)$. We found no difference in the 3-month mortality rate $(21.0 \%$ in the melatonin group v. and $21.4 \%$ in the placebo group; $p=0.9$ ).

In the additional analysis for patients with cognitive impairment at baseline, we found no effect of melatonin on the incidence of delirium (43/104 [41.3\%] for the melatonin group $v$. $37 / 106$ [34.9\%] for the placebo group; $p=0.4$ ).

Table 2: Primary and secondary outcomes

\begin{tabular}{|c|c|c|c|}
\hline \multirow[b]{2}{*}{ Outcome } & \multicolumn{2}{|c|}{ Study group; no. (\%) or median (IQR) } & \multirow[b]{2}{*}{$p$ value } \\
\hline & Melatonin $(n=186)$ & Placebo $(n=192)$ & \\
\hline \multicolumn{4}{|l|}{ Primary outcome } \\
\hline Incidence of delirium & $55(29.6)$ & $49(25.5)$ & 0.4 \\
\hline $\begin{array}{l}\text { Secondary outcomes: patients } \\
\text { with delirium }\end{array}$ & $n=55$ & $n=49$ & \\
\hline Duration of delirium, $d$ & $2(1.0-3.0)$ & $2(1.0-3.0)$ & 0.5 \\
\hline Duration of delirium $>2 \mathrm{~d}$ & $14(25.5)$ & $23(46.9)$ & 0.02 \\
\hline Severe delirium* & $25(45.4)$ & $26(53.1)$ & 0.4 \\
\hline Length of hospital stay, d & $11(6.0-14.5)$ & $11(8.0-17.0)$ & 0.4 \\
\hline Use of antipsychotics, mg & $4.0(1.5-7.5)$ & $5.0(3.8-8.3)$ & 0.2 \\
\hline $\begin{array}{l}\text { Use of benzodiazepines, mg oxazepam } \\
\text { equivalents }\end{array}$ & $63.4(33.4-104.3)$ & $75.0(33.3-131.3)$ & 0.6 \\
\hline Secondary outcomes: all patients & $n=186$ & $n=192$ & \\
\hline Death during admission & $4(2.2)$ & $4(2.1)$ & $>0.9$ \\
\hline Death by 3-mo follow-up & $39(21.0)$ & $41(21.4)$ & 0.9 \\
\hline $\begin{array}{l}\text { Secondary outcomes at } 3 \text { mo: } \\
\text { all patients }\end{array}$ & $n=147$ & $n=151$ & \\
\hline Katz-ADL score & $9.0(5-13)$ & $9.0(5-13)$ & 0.5 \\
\hline Data missing & $3(2.0)$ & $6 \quad(4.0)$ & \\
\hline Cognitive impairment & $87(59.2)$ & $105(66.0)$ & 0.4 \\
\hline
\end{tabular}


Among the patients who experienced delirium, fewer of those receiving melatonin had a delirium episode longer than 2 days $(10 / 43$ [23.2\%] v. 19/37 [51.4\%]; $p=0.01$ ).

\section{Interpretation}

In this multicentre, double-blind, randomized controlled trial involving elderly patients who underwent acute surgery for hip fracture, we observed no effect of melatonin on the incidence of delirium. The per-protocol analysis yielded a similar finding. Also, there was no effect in the subgroup of patients who received the study medication before operation (data not shown). However, among patients who experienced delirium during the study period, a smaller proportion of those receiving melatonin had delirium lasting longer than 2 days. There were no between-group differences in the other secondary outcome measures during the hospital stay or at 3-month follow-up.

These results indicate that, for vulnerable elderly patients with hip fracture, melatonin is not effective in preventing postoperative delirium. Recently, 2 other randomized controlled trials showed substantial decreases in the incidence of delirium among patients receiving melatonin (33\% v. $9 \%^{24}$ and $31 \%$ v. $\left.12 \%{ }^{23}\right)$. The earlier of these 2 studies, ${ }^{24}$ which involved patients undergoing elective surgery, had some methodologic drawbacks due to a lack of clarity about the randomization procedure and blinding methods. The later study ${ }^{23}$ had 2 important differences relative to our study. First, the reduction in incidence of delirium (from $31 \%$ to $12 \%$ ) was achieved in patients admitted to an internal medicine ward; second, the patients were treated prophylactically with a 0.5 -mg dose of melatonin. With regard to the extent of improvement, previous data from the Academic Medical Center (one of the centres in the current study) showed an incidence of delirium of about $50 \%$ among patients with hip fracture. ${ }^{39}$ In the current study, performed on the same wards only 5 years later, the incidence of delirium was about $30 \%$. Improvements in perioperative care by all involved specialties, including supportive care by the geriatric consultation team, likely underlie this remarkable decrease in the incidence of delirium. ${ }^{41,42}$ In view of this relatively low incidence rate in a vulnerable patient group with major precipitating factors (e.g., hip fracture followed by acute surgery), it may be difficult to achieve any additional treatment effect. With regard to the dose of melatonin, we used $3 \mathrm{mg}$ because this dose is frequently used in other studies, including clinical trials of melatonin. ${ }^{43}$
Nevertheless, available information on the effects of melatonin supplementation, on required doses and on duration of supplementation is limited. The higher dose used in our study may have influenced physiologic concentrations during the day and therefore the overall effect. The intervariability of endogenous melatonin levels and the pharmacokinetics of exogenous melatonin might have played a role in the negative results of this study. ${ }^{43}$

The median duration of delirium was similar between the study groups. However, there was some indication that perioperative melatonin supplementation resulted in fewer patients having a long duration ( $>2$ days) of delirium. Any reduction in the time that patients suffer from delirium is important, given that delirious experiences involve very unpleasant feelings, such as fear, anxiety and a feeling of being threatened. Many patients have unpleasant memories and flashbacks. ${ }^{44}$ Also, 1-year outcomes for measures such as global cognition are worse for patients with delirium of longer duration., ${ }^{2,45}$

Melatonin has both chronobiotic and nonchronobiotic properties. It is possible that it resets the sleep-wake cycle through its influence on the biological clock. ${ }^{46}$ Alternatively, it may play a direct role in the pathophysiology of delirium. Delirium is thought to be caused primarily by inflammation of the central nervous system and dopaminergic dysfunction. Melatonin is one of the many anti-inflammatory molecules produced at the sites of lesions during the recovery

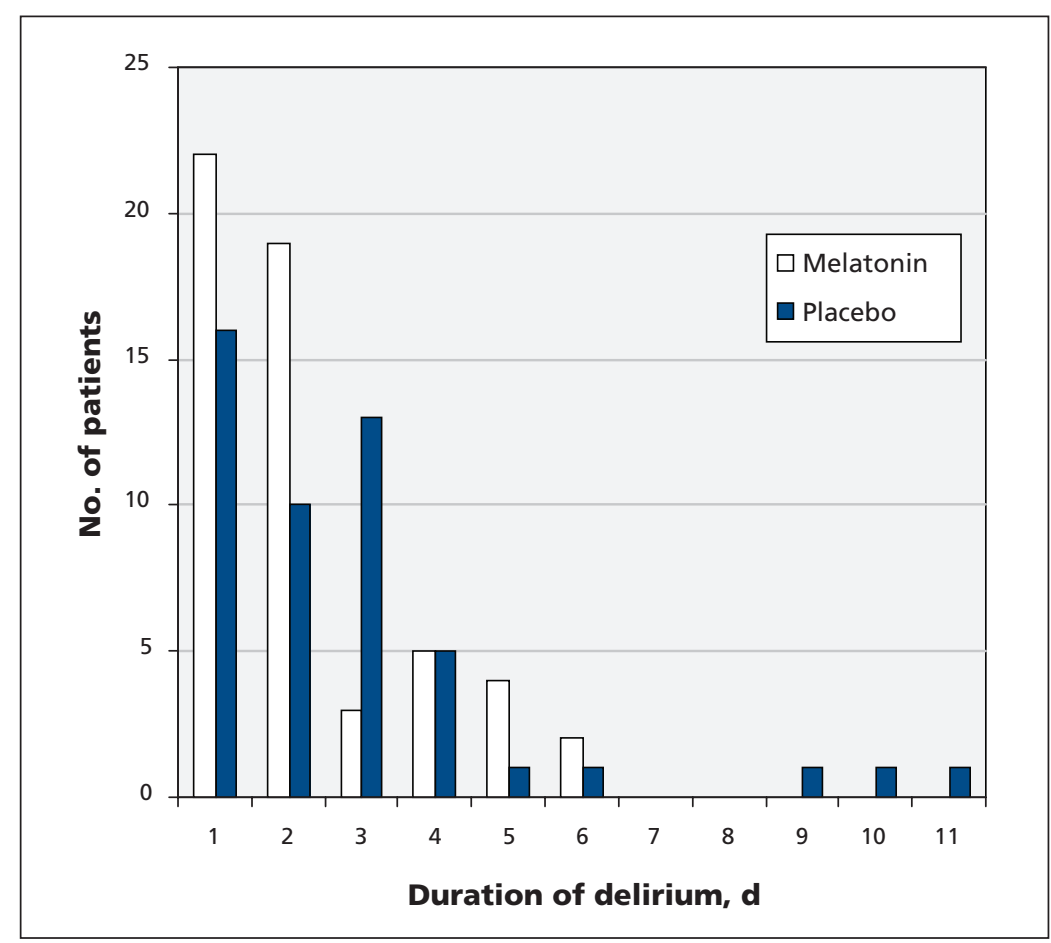

Figure 2: Duration of delirium for patients receiving melatonin or placebo. 
phase of an inflammatory response, and it is involved in the modulation of central dopaminergic functions. ${ }^{17,47-50}$ The fact that these actions require time to become effective after supplementation could underlie our findings that melatonin had no effect on the incidence of delirium but that it could affect duration after a critical period of 2 days. ${ }^{51}$

The main strength of this study was our pragmatic trial approach, with few exclusion criteria. Also, $60.4 \%$ of eligible patients consented to participate, a higher percentage than in any comparable studies involving vulnerable elderly populations. ${ }^{52}$ Therefore, our results have good external validity and can be extrapolated to other populations of patients with hip fracture..$^{53}$ Furthermore, we applied a clear definition of delirium based on the gold standard (DSM-IV) definition. ${ }^{37}$

\section{Limitations}

One limitation of this study was the loss of statistical power because of numerous postrandomization exclusions (see Figure 1). This high rate of attrition is typical of studies involving vulnerable older patients, as the 2 main independent factors related to increased attrition are increasing age and cognitive impairment. ${ }^{54} \mathrm{We}$ initially reached the predefined sample size of 452 patients but were able to analyze data for only 378 patients, which increases the probability of type 2 error. However, given the absence of any treatment effect of melatonin on the incidence of delirium, a much larger sample size would have been necessary to reach statistical significance, and we argue that this would not have resulted in any clinically relevant effect on incidence (i.e., the number needed to treat would be unjustifiably large).

Also, we had planned in advance to express the severity of delirium using the Delirium Rating Scale - Revised 98 score. ${ }^{55,56}$ A trajectory of scores (i.e., to show a pattern of results over several days) would be most informative but proved infeasible because of the relatively short episodes of delirium. Therefore, we instead decided to use the total amount of haloperidol prescribed. Total dose of haloperidol is a clinically more relevant outcome measure, as its adverse effects are more prevalent among patients receiving higher (cumulative) doses. Because the duration of most delirium episodes was 1 or 2 days and our institution's protocol prescribed twice-daily 0.5 -mg doses, we chose a cut-off total of at least $3 \mathrm{mg}$ haloperidol as an indicator of severity.

Another limitation could be that delirium was incorrectly diagnosed in some patients who were drowsy from the surgical anesthesia. However, all patients went first to a recovery room after the operation, so we believe there was an adequate interval between the operation and our observations on the wards to correctly evaluate for presence of delirium.

Furthermore, patients with delirium might have been missed if they had hypoactive delirium. However, our research team was trained to recognize this form of delirium, performed psychiatric evaluations for all patients every day and used subtype measurement tools such as the Delirium Symptom Interview ${ }^{57}$ and the Delirium Motor Subtype Scale ${ }^{58}$ daily to compare their observations.

\section{Conclusion}

Our study has shown that among elderly patients undergoing acute surgery for hip fracture, perioperative melatonin supplementation is ineffective in decreasing the incidence of delirium. However, the proportion of patients with a long-lasting episode of delirium was lower among those who received melatonin, representing a reduced duration of unpleasant delirious experiences for these patients. Future research concerning melatonin and delirium should focus on different patient populations and should compare the potential effects of melatonin head-to-head with antipsychotics in patients with hip fracture who also have delirium. In addition, melatonin supplementation should be investigated in other populations with disturbances of the circadian sleep-wake cycle and a high incidence of delirium, such as patients in the ICU. These additional studies should consider variations in physiologic melatonin levels and different doses of melatonin.

\section{References}

1. Witlox J, Eurelings LS, de Jonghe JF, et al. Delirium in elderly patients and the risk of postdischarge mortality, institutionalization, and dementia: a meta-analysis. JAMA 2010;304:443-51.

2. Saczynski JS, Marcantonio ER, Quach L, et al. Cognitive trajectories after postoperative delirium. N Engl J Med 2012;367:30-9.

3. O'Mahony R, Murthy L, Akunne A, et al. Synopsis of the National Institute for Health and Clinical Excellence guideline for prevention of delirium. Ann Intern Med 2011;154:746-51.

4. Kalisvaart KJ, de Jonghe JF, Bogaards MJ, et al. Haloperidol prophylaxis for elderly hip-surgery patients at risk for delirium: a randomized placebo-controlled study. J Am Geriatr Soc 2005; 53:1658-66.

5. Ballard C, Hanney ML, Theodoulou M, et al. The dementia antipsychotic withdrawal trial (DART-AD): long-term follow-up of a randomised placebo-controlled trial. Lancet Neurol 2009;8:151-7.

6. Brodaty H, Ames D, Snowdon J, et al. A randomized placebocontrolled trial of risperidone for the treatment of aggression, agitation, and psychosis of dementia. J Clin Psychiatry 2003;64:134-43.

7. Antipsychotics, conventional and atypical. Silver Spring (MD): US Food and Drug Administration; 2008. Available: www.fda gov/Safety/MedWatch/SafetyInformation/SafetyAlertsforHuman MedicalProducts/ucm110212.htm (accessed 2012 May 14).

8. Gaudreau JD, Gagnon P, Roy MA, et al. Association between psychoactive medications and delirium in hospitalized patients: a critical review. Psychosomatics 2005;46:302-16.

9. Pandharipande P, Shintani A, Peterson J, et al. Lorazepam is an independent risk factor for transitioning to delirium in intensive care unit patients. Anesthesiology 2006;104:21-6.

10. Meagher DJ, Leonard M, Donnelly S, et al. A longitudinal 
study of motor subtypes in delirium: relationship with other phenomenology, etiology, medication exposure and prognosis. J Psychosom Res 2011;71:395-403.

11. Figueroa-Ramos MI, Arroyo-Novoa CM, Lee KA, et al. Sleep and delirium in ICU patients: a review of mechanisms and manifestations. Intensive Care Med 2009;35:781-95.

12. de Jonghe A, Korevaar JC, van Munster BC, et al. Effectiveness of melatonin treatment on circadian rhythm disturbances in dementia. Are there implications for delirium? A systematic review. Int J Geriatr Psychiatry 2010;25:1201-8.

13. Miyazaki T, Kuwano H, Kato H, et al. Correlation between serum melatonin circadian rhythm and intensive care unit psychosis after thoracic esophagectomy. Surgery 2003; 133:662-8.

14. Shilo L, Dagan Y, Smorjik Y, et al. Patients in the intensive care unit suffer from severe lack of sleep associated with loss of normal melatonin secretion pattern. Am J Med Sci 1999;317: 278-81.

15. Haimov I. Melatonin rhythm abnormalities and sleep disorders in the elderly. CNS Spectr 2001;6:502-6.

16. Olofsson K, Alling C, Lundberg D, et al. Abolished circadian rhythm of melatonin secretion in sedated and artificially ventilated intensive care patients. Acta Anaesthesiol Scand 2004;48: 679-84.

17. de Rooij SE, van Munster BC. Melatonin deficiency hypothesis in delirium. a synthesis of current evidence. Rejuvenation Res 2013; 16:273-8.

18. Balan S, Leibovitz A, Zila SO, et al. The relation between the clinical subtypes of delirium and the urinary level of 6-SMT. $J$ Neuropsychiatry Clin Neurosci 2003;15:363-6.

19. Hanania M, Kitain E. Melatonin for treatment and prevention of postoperative delirium. Anesth Analg 2002;94:338-9.

20. Shigeta H, Yasui A, Nimura Y, et al. Postoperative delirium and melatonin levels in elderly patients. Am J Surg 2001;182:449-54

21. Sher L. Postoperative delirium, plasma melatonin, and light Med Hypotheses 2001;56:411-2.

22. Uchida K, Aoki T, Ishizuka B. Postoperative delirium and plasma melatonin. Med Hypotheses 1999;53:103-6.

23. Al-Aama T, Brymer C, Gutmanis I, et al. Melatonin decreases delirium in elderly patients: a randomized, placebo-controlled trial. Int J Geriatr Psychiatry 2011;26:687-94.

24. Sultan SS. Assessment of role of perioperative melatonin in prevention and treatment of postoperative delirium after hip arthroplasty under spinal anesthesia in the elderly. Saudi $J$ Anaesth 2010;4:169-73.

25. Hatta K, Kishi Y, Wada K, et al. Preventive effects of ramelteon on delirium: a randomized placebo-controlled trial. JAMA Psychiatry 2014;71:397-403.

26. de Jonghe A, van Munster BC, van Oosten HE, et al. The effects of melatonin versus placebo on delirium in hip fracture patients: study protocol of a randomised, placebo-controlled, double blind trial. BMC Geriatr 2011;11:34.

27. Good clinical practice. European Commission; 1990 [revised 1996]. Available: http://ec.europa.eu/health/files/eudralex/vol-10 /3cclaen_en.pdf (accessed 2014 Aug. 25).

28. Koch BC, Nagtegaal JE, Kerkhof GA, et al. Circadian sleepwake rhythm disturbances in end-stage renal disease. Nat Rev Nephrol 2009;5:407-16.

29. Nickkholgh A, Schneider H, Sobirey M, et al. The use of highdose melatonin in liver resection is safe: first clinical experience. J Pineal Res 2011;50:381-8.

30. Good manufacturing practice (GMP) guidelines/inspection checklist. Silver Spring (MD): US Food and Drug Administration; 1997 [updated 2008]. Available: www.fda.gov/cosmetics/guidance regulation/guidancedocuments/ucm2005190.htm (accessed 2013 June 6).

31. Weinberger M, Samsa GP, Schmader K, et al. Comparing proxy and patients' perceptions of patients' functional status: results from an outpatient geriatric clinic. J Am Geriatr Soc 1992;40:585-8.

32. Folstein MF, Folstein SE, McHugh PR. "Mini-mental state." A practical method for grading the cognitive state of patients for the clinician. J Psychiatr Res 1975;12:189-98.

33. Jorm AF. A short form of the Informant Questionnaire on Cognitive Decline in the Elderly (IQCODE): development and cross-validation. Psychol Med 1994;24:145-53.

34. de Jonghe JF. Differentiating between demented and psychiatric patients with the Dutch version of the IQCODE. Int J Geriatr Psychiatry 1997;12:462-5

35. Jorm AF. The Informant Questionnaire on Cognitive Decline in the Elderly (IQCODE): a review. Int Psychogeriatr 2004;16: 275-93.

36. Charlson ME, Pompei P, Ales KL, et al. A new method of classifying prognostic comorbidity in longitudinal studies: development and validation. J Chronic Dis 1987;40:373-83.
37. Diagnostic and statistical manual of mental disorders. Fourth edition, text revision. Washington: American Psychiatric Association; 2000.

38. Schuurmans MJ, Shortridge-Baggett LM, Duursma SA. The Delirium Observation Screening Scale: a screening instrument for delirium. Res Theory Nurs Pract 2003;17:31-50.

39. van Munster BC, Korevaar JC, Zwinderman AH, et al. Timecourse of cytokines during delirium in elderly patients with hip fractures. J Am Geriatr Soc 2008;56:1704-9.

40. Cole MG, Primeau F, McCusker J. Effectiveness of interventions to prevent delirium in hospitalized patients: a systematic review. CMAJ 1996;155:1263-8.

41. Chen CC, Lin MT, Tien YW, et al. Modified hospital elder life program: effects on abdominal surgery patients. J Am Coll Surg 2011;213:245-52.

42. Inouye SK, Bogardus ST Jr, Charpentier PA, et al. A multicomponent intervention to prevent delirium in hospitalized older patients. N Engl J Med 1999;340:669-76.

43. Vural EM, van Munster BC, de Rooij SE. Optimal dosages for melatonin supplementation therapy in older adults: a systematic review of current literature. Drugs Aging 2014;31:441-51.

44. O'Malley G, Leonard M, Meagher D, et al. The delirium experience: a review. J Psychosom Res 2008;65:223-8.

45. Pandharipande PP, Girard TD, Jackson JC, et al. Long-term cognitive impairment after critical illness. N Engl J Med 2013;369: 1306-16.

46. Brzezinski A. Melatonin in humans. N Engl J Med 1997;336: 186-95.

47. Maldonado JR. Pathoetiological model of delirium: a comprehensive understanding of the neurobiology of delirium and an evidence-based approach to prevention and treatment. Crit Care Clin 2008;24:789-856.

48. Zisapel N, Laudon M. Dopamine release induced by electrical field stimulation of rat hypothalamus in vitro: inhibition by melatonin. Biochem Biophys Res Commun 1982;104:1610-6.

49. Pandi-Perumal SR, Bahammam AS, Brown GM, et al. Melatonin antioxidative defense: therapeutical implications for aging and neurodegenerative processes. Neurotox Res 2013;23:267-300.

50. Maclullich AM, Ferguson KJ, Miller T, et al. Unravelling the pathophysiology of delirium: a focus on the role of aberrant stress responses. J Psychosom Res 2008;65:229-38.

51. Cronin AJ, Keifer JC, Davies MF, et al. Melatonin secretion after surgery. Lancet 2000;356:1244-5.

52. Gamberini M, Bolliger D, Lurati Buse GA, et al. Rivastigmine for the prevention of postoperative delirium in elderly patients undergoing elective cardiac surgery - a randomized controlled trial. Crit Care Med 2009;37:1762-8.

53. Van Spall HG, Toren A, Kiss A, et al. Eligibility criteria of randomized controlled trials published in high-impact general medical journals: a systematic sampling review. JAMA 2007;297:1233-40.

54. Chatfield MD, Brayne CE, Matthews FE. A systematic literature review of attrition between waves in longitudinal studies in the elderly shows a consistent pattern of dropout between differing studies. J Clin Epidemiol 2005;58:13-9.

55. Trzepacz PT, Baker RW, Greenhouse J. A symptom rating scale for delirium. Psychiatry Res 1988;23:89-97.

56. de Rooij SE, van Munster BC, Korevaar JC, et al. Delirium subtype identification and the validation of the Delirium Rating Scale - Revised-98 (Dutch version) in hospitalized elderly patients. Int J Geriatr Psychiatry 2006;21:876-82.

57. Albert MS, Levkoff SE, Reilly C, et al. The delirium symptom interview: an interview for the detection of delirium symptoms in hospitalized patients. J Geriatr Psychiatry Neurol 1992;5:14-21.

58. Meagher D, Moran M, Raju B, et al. A new data-based motor subtype schema for delirium. J Neuropsychiatry Clin Neurosci 2008;20:185-93.

Affiliations: Department of Internal Medicine, Geriatrics Section (de Jonghe, van Munster, de Rooij), Department of Surgery, Trauma Unit (Goslings), Department of Orthopedic Surgery (Kloen), Department of Internal Medicine (Levi) and Clinical Research Unit (de Haan), Academic Medical Center, University of Amsterdam, Amsterdam, The Netherlands; Department of Geriatrics (van Munster), Gelre Hospitals, Apeldoorn, The Netherlands; Department of Geriatrics (van Rees), Department of Orthopaedic Surgery (Wolvius) and Department of Surgery (van Velde), Tergooi Hospitals, Hilversum, The Netherlands.

Contributors: Sophia de Rooij developed the idea for the study and obtained funding. Annemarieke de Jonghe was responsible for project organization. Sophia de Rooij and Bar- 
bara van Munster contributed to study conception and design. Annemarieke de Jonghe, Carel Goslings, Peter Kloen, Romuald van Velde, Reinder Wolvius and Carolien van Rees contributed to acquisition of data. Annemarieke de Jonghe, Rob de Haan, Barbara van Munster, Marcel Levi and Sophia de Rooij contributed to analysis and interpretation of data. Sophia de Rooij, Annemarieke de Jonghe and Barbara van Munster were principally responsible for drafting this manuscript. All authors contributed substantially to drafting the article or revising it critically for important intellectual content and gave final approval of the version to be published. Annemarieke de Jonghe had full access to all of the study data (reported and unreported) and takes responsibility for integrity of the data and accuracy of the analysis. All authors agree to be accountable for all aspects of the work in ensuring that questions related to the accuracy or integrity of any part of the work are appropriately investigated and resolved.

Members of the Amsterdam Delirium Study Group: Sophia de Rooij, Barbara van Munster, Annemarieke de Jonghe and Rikie Scholtens.

Funding: This study was funded by an unrestricted grant from the Dutch National Program of Innovative Care for vulnerable older persons (grant no. 311020301). This program is operated by ZonMw, a Dutch institute that funds health research and stimulates the use of knowledge to help improve health and health care in the Netherlands. Apart from the funding, this organization had no further involvement in the study (data collection, analysis or reporting of results, or preparation for publication). This investigatorinitiated study was not sponsored by the manufacturer of Circadin or any other melatonin products.

Data sharing: All data are available to be shared. Requests should be sent to, and will be judged by, the corresponding author.

Acknowledgements: The authors would like to thank the following people who contributed to data collection in the Academic Medical Center: José Popma, Annemiek van Helden, Isabella Makkinje, Juliette Parlevliet, Nathalie van der Velde, Orla Smit, Nienke Brendel, Marike van Hengel and Simone Jong. They also thank those who contributed to data collection in Tergooi Hospitals: Nicolette de Vries, Mardou van Leeuwenstijn and Harm van Orden. These contributions to the study were performed in the course of their regular duties. The authors also thank Esmee Vural, of the Academic Medical Center, who was responsible for confirmation of data entry. 